\title{
Role of FACTS devices in improving penetration of renewable energy
}

\begin{abstract}
In recent years, the renewable or green energy has been a major focus globally. Moreover, this energy can be like solar and wind which have been increasingly implemented worldwide. However, the adoption of the green energy is still low due to high technological costs and the associated power system issues. The effectiveness of Flexible Alternating Current Transmission System (FACTS) devices in problems that are related to the power systems has been tremendous. Although, there are still relatively limited works on incorporating FACTS devices into green energy plants. The progress of the FACTS application in renewable energy plants has been reviewed in this work. In addition, it has been discovered that FACTS plays an equally effective role in improving the power system quality of green energy plants especially in wind and solar plants. The rate of penetration of green energy could be accelerated if applications of FACTS devices in renewable energy plants were to be more widely adopted.
\end{abstract}

Keyword: FACTS; Power quality; Renewable energy 